chätennachweis und Blutuntersuchung sind von unschätzbarem Nutzen nicht nur für den Einzelfall, sondern auch für die allgemeine Bedeutung der Syphilis als Volkskrankheit geworden. Besonders aber im Salvarson hat uns Ehrlich in bewundernswerter, langwieriger und methodischer Forscherarbeit eine neue Waffe zur Abtötung der Syphiliserreger geschmiedet und somit einen sicheren Weg zur Heilung der Syphitis gebahnt. Durch diese Entdeckung hat die Erkenntnis über die Gefahren, die die Syphilis dem einzelnen und dem Volkskörper bringen kann, unendlich gewonnen und die Krankheit auch vieles von ihrem unheimlichen Charakter verloren. Die Heilung der Syphilis ist heute eine viel sichere und sehnellere geworden, wenn auch das Ideal, das Ehrlich vorgeschwebt hat, durch eine einzige Injektion eine endgültige Heilung zu erzielen, noch nicht erreicht worden ist. Hoffen wir, daß das bisher errichtete Gebäude mit der Erreichung auch dieses Zieles baldigst gekrönt wird. Möge auch die Entdeckung jenes Idealpräparates demjenigen gelingen, der unermüdlich weiter arbeitet, dem die Syphilisforschung schon so auperordentliches zu danken hat: Paul Ehrlich!

\section{Zur Salvarsanfrage.}

Von Marineoberstabsarzt $D_{r}$. Gennerich, Kiel.

Dieser Tage durcheilte die Tagespresse die überraschende Kunde, daß ein Berliner Polizeiarzt namens Dreuw dem Reichsgesundheitsamt eine Denkschrift überreicht habe, um ein Reichsverbot gegen Salvarsan zu erwixken, und zwar mit dem Hinweis, daB das Mittel vor ausreichender Erprobung in den Verkehx gelangt sei, und sich später als ein Mittel entpuppt habe, das Leben und Gesundheit des Patienten auf das Schwerste geführde. Dr. Dreuw hat ferner einen Berichterstatter dahin informiert, daß die Unglücksfalle nach Salvarsan nicht etwa auf falscher Anwendung beruhten, sondern auch bei einwandfreier Applikation zutage träten. Neben ca, 275 Todesfällen sei eine große Anzahl von Erblindungen, Läbmungen und Taubheit als Folge von Salvarsanbehandlung bekannt geworden. Alle diese Störungen seien auf den enormen Arsengehalt des Präparates zurückzuführen, das mehr als das Zehnfache der für Arsen maßgebenden Dosis betrage. Ein derart gefährliches Mittel müsse aber, selbst wenn es in andern Fällen Heilung biete, von der Bildfläche verschwinden, um so mehr wenn wegen der ungenügenden Erprobung nicht einmal eine Heilwirkung feststehe. Seine Ansichten würden geteilt von der Straßburger HautKlinik.

Es ist nicht das erste $\mathrm{Mal}$, daß Nachrichten iber die Wirksamkeit des Salvarsans in ausgiebimem Mabe in die Presse gelangen. Im Anfange der Salyarsanära wurden bekanntlich auBerordentlich gute Erfolge über die Heilwirkung des Ehrlich-Hata 606 berichtet, Beobachtungen, welche sich aus der außerordentlich günstigen Einwirkung des Präparates auf die sichtbaren Erscheinungen der Syphilis ergaben. Es zeigte sich aber sehr bald, zunächst aus den. Nachprüfungen mittels der Wassermannschen Serumreaktion, später auch aus der Wiederkehr klinischer Frscheinungen, daß sieh die ursprünglichen Erwartungen, die sich bei der Erprobung des Mittels im Tierexperiment ergeben hatten, bei Anwendung vereinzelter Salvarsaninjektionen nicht verwirklichen ließen. Bereits hierdurch wurde das Interesse zu der neuen Behandlung erheblich eingeebbt. Es entstanden überdies aber dem Salvarsan noch heftige Gegner, sobald es sich bei der weiteren Anwendung des Präparates gezeigt hatte, daB Störungen zutage traten, welche man bei der früher üblichen Behandlungsmethode nur wenig oder gar nicht gesehen hatte und sobald femer die Technik der Behandlung in ein bis dahin therapeutisch nur wenig ausgenutztes Gebiet, nämlich in das der intravenösen Einverleibung, übergriff.

Es bedurfte natürlich geraumer Zeit, um die Teistungsfähigkeit der neuen Behandlungsmethode kennen zu lernen, und um auch ein Urteil zu gewinnen über die Herkunft ungünstiger Nebenerscheinungen und ubber die Möglichkeit, ihnen zu begegnen. Es wäre aber zweifellos ein Dnrecht von Ehrlich gewesen, wenn er die Freigabe des Heilmittels noch länger verzögert hätte. Man hätte dadurch eine unendlich große Zahl von Syphiliskranken von den großen Segnungen der Behandlungsmethode ausschalten müssen.

Ohne Salvarsan wären jedenfalls bei uns eine ganze Anzahl ron Fällen mit schwerer nervöser Syphilis und GefäBsyphilis und auch mehrere Fälle der sogenannten bösartigen Syphilis, welche auf Quecksilber alle nicht mehr reagierten und sich in einer trostlosen Verfassung befanden, einem sicheren Tode in kürzester Frist ausgeliefert gewesen.

Welche Ziele die Gegner der Salvarsanbehandlung mit ihren Angriffen verfolgen, ist uns nicht recht verständlich, weil wir von den Ursachen der Vergiftungsvorgänge und auch von der Leistungsfähigkeit der neuen Behandlung ganz andere Beobachtungen besitzen, als sie auf der gegnerischen Seite geltend gemacht werden.

Vor allem haben wir hinsichtlich der Vergiftungserscheinungen die Erfahrung gemacht, daB sie auf Unvollkommenheiten in der Behandlung zurückzuführen sind, die man bei ausreichender Erfahrung auf dem Gebiet der neven Therapie gänzlich ausschließen oder auf ein belangloses Minimum zurückführen kann.

Es muß jedoch unter allen Tmständen den Gegnern der Salvarsanbehandlung das Recht eingeräumat werden, ihre ungünstigen Behandlungserfolge durch medizinische Fachzeitschriften ärtlichen Kreisen zugänglich zu machen. Es dưfte 
sich wohl jede Zeitschrift hierzu verstehen, so lange die Autoren objektiv bleiben und auch den Anschein einer persönlichen Polemik vermeiden.

Die bei uns übliche kombinierte Salvarsanbehandling verläuft heute in derart reaktionslosen Bahnen, daß seit vielen Monaten auch nicht die geringste Störung mehr beobachtet wurde.

Es hat sich ferner mit der zunehmenden Erkenntnis von der Wirkungsweise des Salvarsans bei uns allmählich ein Behandlungsplan für die einzelnen Syphilisstadien herausgebildet, welcher uns jetzt in $95 \%$ der Fälle einen einwandfreien Verlauf gebracht hat. Auf die Bedeutung dieser Tatsache wollen wir unten noch in Kürze einrehen.

An sich ist die Einverleibung einer intravenösen Salvarsaninjektion keineswegs als eine besondere Leistung anzusehen.

Wie aber 2 Welten rerschieden, so kann sich auch die Technik der Zubereitung der Salvarsanlösung und die Ausgestaltung des einzelnen Behandlungsplanes in der Hand des einzelnen Therapeuten verschieden gestalten.

In diesem Sinne ist die Salvarsanbehandlung cine ärztliche Kunst, die jedoch bei der nötigen Fortbildung jedem einzelnen zugänglich ist.

Leider war es von vornherein nicht unter allen Umständen möglich, die Gefahrzonen der Behandlung mit absoluter Sicherheit auszuschalten, besonders wenn das vorhandene Syphilisstadium eine recht gründliche Behandlung exforderte. Dazu war uns, wie bereits oben angedeutet, das Gebiet einer ausgedehnten intravenösen Behandlung, wie auch das der modernen Chemotherapie zunächst noch zu fremd.

Es kann daher m. E. dem Ansehen eines tüchtigen Arztes keineswegs sehaden, wenn er bei den Fortschritten der Technik der Erkenntnis Raum gibt, daß nach den Unglücksfüllen der ursprüunglich üblichen Behandlungsweise technische Unzulänglichkeiten, sei es Fehler der Salvarsanlösung oder sei es individuelle Utberdosierung, maßgebend gewesen sind.

Auch bei uns ist der Ausbau der Therapie nicht ohne Wehen vor sich gegangen. Wir haben unter reichlich 1200 Fällen nur 2 Todesfälle beobachtet, von denen jedoch nur einer direkt mit der Salvarsananwendung im Zusammenhange steht. In diesem Ealle waren wir bei der Neuaufnahme der Neosalvarsanbehandlung einem von andrer Seite empfohlenen Behandlungsplane gefolgt.

Nach den bisherigen Beobachtungen war jedenfalls die Mortalität nach Salvarsan die gleiche, wie früher bei der alleinigen Quecksilberbehandlung.

Wir haben jedoch die Ủberzeugung gewonner, daß wir bei der hentigen Entwicklung der Technik und bei dem jetzigen Stande unserer Erfahrungen durchaus in der Juage sind, auch die geringsten Behandlungsstörungen zu vermeiden.
Wie mühsam aber der Fortsehritt war, wieviel Unuhe und Sorge der allmähliche Ausbau der Behandlung verursacht hat, kann nur der ermessen, der an ihr aktiven Anteil genommen und zugleich auf das Ziel einer ausreichenden Behandlung in allen Fällen hingearbeitet hat.

Die Dreuwschen Mitteilungen fallen aber heute in eine Zeit hinein, wo sich fast alle gröBeren Behandlungsstätten die Fortschritte der Salvarsanbehandlung zu eigen gemacht haben und auf dem Boden einer reaktionslosen Behandlung operieren lönnen. Es gibt für gewöhnlich nur Fiebersteigerung auf die erste Salvarsaninjektion in unvorbehandelten ganz frischen Fällen, was aber für die weitere Fortfïhrung der Behandlung belanglos ist.

Der Schaden der Dreuwschen Nachrichten in der Tagespresse liegt zunächst in einer Beunruhigung des Publikums.

Aber auch manche Veröfentlichungen in der Fachpresse stiften dadurch Schaden, daß sie über die Giftigkeit des Salvarsans jammern und alle Ratschläge, die hinsichtlich der Verbesserung der Technik und zur Durchführung einer gemäßigten und individuellen Dosierung gegeben werden, als verderbliche Versuche und Täuschungsmanöver hinstellen, um über die Giftigkeit des Salvarsans hinwegrukommen.

Daß bei einer mangelhaften Behandlungstechnik außer zahlreichen Störungen auch sehr mangelhafte Resultate herauskommen, bedarf keiner Ausführung.

Eine ausreichende Behandlung ist bei einem derartigen Vorgehen absolut ausgeschlossen.

Utber die Bedeutung der Behandlungserfolge nach Salvarsanbehandlung kann man natürlich nur ein Urteil abgeben, wenn man auf Grund von Dauerbeobachtungen, wozu sich unser Krankenmaterial bej der Marine wegen ihrer zahlreichen Kapitulanten besonders gut eignet, in der Lage ist, über die Wirksamkeit der früher üblichen Quecksilberbehandlung sich zu äußern und sich überhaupt über das Verhalten der syphilitischen Infektion unter den Einflüssen del verschiedensten Behandlungsweisen auskennt.

Die Angaben Dreuws, dal die Syphilis bei seinen Prostituierten durch 1-2 Quecksilberliuren ausgeheilt sei, was er durch Befragen dieser Personen festgestellt habe, wird in Fachkreisen nur allgemeinem Kopfschütteln begegnen.

Wir müssen uns jedenfalls in jeder Beziehung dem Neißerschen Standpunkte anschließen, welcher 6-8 planmäBig intermittierende Quecksilberkuren im Verlaufe von $4-5$ Jahren fordert.

Die Dreuwschen Angaben, daß eine Syphilis in 2-3 Jahren für gewöhnlich ausheilt, müssen wir unbedingt als unrichtig zurückweisen. Sie können nur dadurch zustande kommen, daB Dreuw die Späterscheinungen der Syphilis nicht mehr zu sehen bekommt, die erst eintreten, wenn die Prostituierte längst nicht mehr ihrem Gewerbe nachzugehen imstande ist und mit syphilitischen $\mathrm{Ver}$ - 
änderungen am Herzen, as den Gefäßen, am Zentralnervensystem oder an anderen Organen in ganz andere ärztliche Hände gelangt, als die des Dermatologen.

Um die Häufigkeit einzelner Folgezustände der Syphilis zu erwäbnen, möchte ich nur eine kürzlich erschienene Arbeit von Marinestabsarzt. Ahrens erwähnen, der in den letzten 10 Jahren allein 96 Fälle (wobei die Statistik noch als unvollkommen bezeichnet wird) von Gehirnerweichung bei der Marine gezählt hat, für die höchstens 1200 Syphilisfälle in Frage kommen. Gehïnsyphilis und Rückenmarksschwindsucht und syphilitische GefäBerkrankungen des Gehims sind aber wohl kaum viel seltener. Ich habe allein in den letzten Jahren wenigstens 100 Fälle der verschiedensten Stadien von Hirn- und Rückenmarkssyphilis mit Salvarsan behandelt, über die ich bereits zum Teil berichtet habe. Alle diese Krankheitszustände kommen aber bekanntermaßen meist dann erst zur ärztlichen Diagnose, wenn der erste Therapeut der frischen Syphilis des einzelnen Individuums dieses längst aus den Augen verloren hat.

Die modernen Anschauungen von dem Verlauf der Syphilis haben unter einer ausgiebigen $\mathrm{An}$ wendung der serologischen Nachbeobachtung und Liquorkontrolle ein wesentlich anderes Aussehen als früher gewonnen. Vor allem wissen wir heute, daB das Ausbleiben von syphilitischen Krankheitserscheinungen in den ersten Jahren der Syphilis mit einer Heilung des Leidens auch nicht das geringste $\mathrm{zu}$ tun hat.

Gerade diejenigen Syphilisfälle, welche wegen Fehlens von Erscheinungen sehr milde oder gar nicht behandelt worden sind, stellen das bei weitem größere Kontingent der Spätsyphilisfälle. Bereits 1908-1910 konnten wir bei planmäBiger inter. mittierender Quecksilberbehandlung nachweisen, daß die positive Serumreaktion gleich schwer bei Luesfällen zu beseitigen war, gleichgültig ob sicht bare Erscheinungen bestanden oder fehlten.

Der Grund für das Auftreten syphilitiseher Erscheinungen liegt überhaupt lediglich im Rückgange der syphilitischen Allgemeindurchseuchung des Organismus, Es ist dabei einerlei, ob dieser Rückgang. durch spezifische Behandlung oder im Spätstadium der Syphilis durch die sich um das 3.--- - Jahr der Erkrankung allmählich entwickelnden spezifischen Immunvorgänge des $\mathrm{Or}_{\mathrm{r}}$ ganismus zustande kommit. Die Krankheitserreger haben stets das Bestreben, das verlorengegangene Terrain wieder zu gewinnen, dabei kommt es zu Erscheirungen.

Auf der andern Seite besteht für eine wenig oder gar nicht behandelte Syphilis gar kein Grund zu neuen Schüben und Frscheinungen; der ubiquitäre Charakter des Leidens besteht fort und schützt daher vor der Wiederkehr sichtlicher neuer Ausbreitungszeichen der Syphilis.

Hierauf berwhen auch die Erfolge der Kurpfuscher, denn es gehört in einer großen Anzahl vou Syphilisfallen eben nichts dazu, um sie von vornherein in einen vorborgenen Verlauf ihres Leidens hineinzudrängen.

Besondere Verlaufseigentümlichkeiten der Syphilis werden ferner noch diktiert von einer frühzeitigen. Immunkörperbildung, bei der die Art des Virus eine gewisse Rolle spielt, und schlieBlich auch von dem Grade der Schädigung, den erentuell die Krankheitserreger bei guten Turen erfahren.

Nur die Form der rückfäligen syphilitischen Erscheinungen ist verschieden, je nachdem dio Einschränkung der Erreger durch die Therapie in den frischeren Stadien oder durch die Immunvorgänge im Spätstadium verursacht worden ist.

Im ersten Falle kommt es noch zu den gewöhnlichen, meist harmlosen, aber sehr leicht ansteckenden Sekundärerscheinungen, weil dem Erreger das verlorene Terrain noch zugänglich ist, im zweiten Falle bleibt es aber bei umschriebenen Herdsymptomen, weil die vom Organismus selbst gebildeten Immunvorgänge gegen neue Allgemeinausbreitungen eine ziemlich festgefügte Schranke bilden. Die Immunvorgänge des Körpers also sind es, die dem dritten Stadium der Syphilis ihr besonderes Gepräge geben. Die Erscheinungen dieses Stadiums sind aber gerade dadurch so besonders bösartig und hartnäckig (virulent), weil den Erregern der Weg zu der gutartigen Allgemeinausbreitung durch die Immunvorgänge verlegt wird.

Die Dreuwschen Angaben sind daher zu einer verhängnisvollen Irreführung des Publikums geeignet, weil sie bei diesem den Findruck erwecken können, als ob die von Dreuw angegebene Quecksilberbehandlung imstande wäre, die Patienten von ihrer Syphilis zu befreien.

Derartigen Behauptungen müssen wir jedenfalls ein ganz energisches Veto entgegensetzen, denn unsere Erfahrungen erweisen unzweifelhaft, daB das Gros derart behandelter Fäle einem sehr trüben Schicksal entgegensieht.

Auf der soeben erörterten biologischen Basis wird es verständlich, weshalb früher so viele Autoren bei einer häufigen Quecksilberbehandlung über vermehrte Krankheitsrückfälle berichten; es wird uns aber vollends klar, weshalb heute besonders bei einer oberflächlichen Salvarsanbehandlung von einigen Autoren über eine Zunahme der Krankheitsrückfälle berichtet wird. Ravaut bezeichnet sie häufiger als bei der Quecksilberbehandlung, er sah sie zu $80 \%$ im ersten Jahre.

Auch können wir bei dieser Gelegenheit ein Wort über die Entstehung der nervösen Störungen und Lähmungen nach Salvarsan einflechten.

Durch die leichte Finschränkung der syphilitischen Allgemeindurchseuchung des Organismus durch Salvarsan, was sich durch den schnellen, im Anfang der Salvarsanbehandlung so wundersam erscheinenden Rückgang der klinischen Erscheinungen und die beschleunigte Einwirkung auf die positive SR kundtat, wird in extremster Weise 
bei frischer Syphilis die Grundlage zur Ruickfallbildung erzeugt. Die frische Allgemeinausbreitung ist einmal noch leicht zugänglich, andrerseits fehlen bei mäßiger Behandlung irgendwelche Entwicklungshemmungen.

Es ist nun für jedermann leicht verständlich, dal die Rückfallbildung in erster Linie von den wenig oder gar nicht geschädigten Infektionsherden ausgehen muß. Diese befinden sich aber in allererster Linie in der Gehirn- und Rückenmarksflüssigkeit, wo das Salvarsan nachgewiesenermaßen am allerwenigsten hingelangt. Auf die in anatomischen Verhältnissen beruhende verschlechterte Diffusionsfähigkeit des Salvarsans in die Hirnflüssigkeit will ich hier aber nicht näher eingehen. Daß aber Hindernisse für ein von der Blutbahn aus wirkendes Arzneimittel vorhanden sind, wird man leicht ermessen können, wenn man sich der dickflüssigen Beschaffenheit des Blutes und der rein wässerigen Eigenschaft der Hirnflüssigkeit erinnert.

Die heftige Neuentwicklung der kräftig erhaltenen Spirochätenherde in der Hirn- und Rückenmarksflüssigkeit ist nun die Ursache einer syphilitischen Hirnhautentzündung, die sich in erster Linie auf die durch die Hirnhaut hindurchgehenden Hirnnerven überträgt; daher also die Lähmungs- und Ausfallerscheinungen an so wichtigen nervösen Organen wie Auge und Ohr.

Wir haben diese Störungen an Seh- und Gehörnerven bei unserer Behandlung überhaupt nie gesehen. Nux einmal zeigte sich im Anfange unserer Therapie eine vorübergehende Lähmung an einem Gesichtsnerven (facialis), die aber bei sofortiger energischer Salvarsanbehandlung wieder zurückging, so daß der Fall dienstfähig entlassen werden konnte.

Wenn diese schweren Lähmungen, wie Dreuw angibt, auf die arsenschädigende Wirkung des Salvarsans zurückgeführt werden müßten, so wären doch wohl bei kräftigerer Behandlung, wie wir sie ausgeübt haben, diese Lähmungserscheinungen in erster Linie zu erwarten gewesen.

Genau wie hier die Dreuwschen Angaben nicht mit unseren Erfahrungen in Einklang zu bringen sind, so ist es auch dasselbe mit der angeblichen Ursache der Arsenintoxikation nach SalvarsanbehandJung.

DaB es sich bei den Vergiftungserscheinungen nach Salvarsanauwendung um Arsenwirkungen handelt, wird schwerlich von Ehrtich selbst, noch von den Anhängern der Salvarsanbehandlung irgendwie bezweifelt. Eine reine Arsenwirkung liegt allerdings nicht vor, weil es noch in keinem einzigen Falle nach einer Salvarsanintoxikation zu Störungen am Sehnerven oder anderen Nerven gelrommen ist.

Es ist aber ein gewaltiger Unterschied, ob das Salvarsan an sich bei sachgemäßer Anwendung arsentoxische Eigenschaften besitzt, oder ob diese erst durch Umstände wachgerufen werden, die wir zu vermeiden gelernt haben.
Nach unseren Beobachtungen ist es nun ganz zweifellos, dab das Salvarsan an sich, d. h. in unveränderter Konstitution keine Arsenwirkungen hervorruft. Sie beruhen vielmehr auf einer Zersetzung des Salvarsans.

Dank der intensiven und auBerordentlich aufopfernden Arbeit einer ganzen Anzahl von Autoren, die sich um die Klärung der Salvarsantodesfälle verdient gemacht haben (Wechselmann, Emery, Scholtz, Hoffmann, Dreyfuß, Zieler, Marschalko, Schreiber u. a.) sind wir schon seit längerer Zeit in der glücklichen Lage, die beiden Entwicklungsgänge der Salvarsanzersetzung und damit der Intoxikationen scharf unterscheiden $\mathrm{zu}$ können. In einem Falle erfolgt die Zersetzung des Salvarsans im Glasgefäß noch vor der Injektion, im 2. Falle durch Überschreitung der erträglichen Dosis.

Die chemische Veränderung des Salvarsans im GlasgefäB geschieht einmal durch Oxydation mit dem Luftsauerstoff und zweitens durch das Vorhandensein fremder Bestandteile in der Salvarsanflüssigkeit. Es handelt sich um Kupfer, Blei und Silikate, sie gelangen in die Flüssigkeit hinein durch Gebranch eines unzweckmäBigen Destillierapparates und die Verwendung schlechter Glassorten. Das Neosalvarsan ist von ihnen am stärksten bedroht. Diese Art der Intoxykation setzt prompt ein.

Ist indessen eine Überdosierung oder eine Anhäufung von Salvarsan infolge eines zu kleinen Injektionsintervalles erfolgt, so setzen die stürmischen Vergiftungserscheinungen gewöhnlich erst nach mehrtägiger Pause je nach der Größe der Überdosierung ein. Es ist dies ein ungemein charakteristischer Befund. Die Nieren sind stets nur zur Ausscheidung einer bestimmten, aber je nach der vorausgehenden Inanspruchnahme wechselnden Menge von Salvarsan befähigt. Infolge zu hoher Dosierung verbleibt daher ein Quantum Salvarsan im Kreislauf, bzw, im Körper, ohne rechtzeitig zur Ausscheidung gelangen zu können. Zu einer Funktionsverminderung der Nieren hinsichtlich der SalvarsanausstoBung gehört aber nicht regelmäßig auch eine sonstige qualitative oder quantitative Funktionseinschränkung.

Das im Kreislauf nachgewiesenermaßen zurückgehaltene Salvarsan unterliegt aber natürlich den oxydierenden Einflüssen der Sauerstoffträger des Blutes, so dab es schlieBlich zu einer Zersetzung kommt, bei der die Arsenkomponente des Salvarsans frei wird und dann imstande ist, ihre schädlichen Eigenschaften zu entwickeln.

Die Ausscheidungsbedingungen für Salvarsan sind besonders geschädigt, wenn organische Verunreinigungen der Injektionsflüssigkeit beigemengt sind. Diese Vermeidung des organischen Wasserfehlers ist absolut gleichbedeutend und ebenso wichtig, als die bekannte Gegenanzeige gegen Salvarsanbehandlung, die im Vorhandensein akut fieberhafter Komplikationen liegt, und sei es auch nur in einer frischen Hodenentzindung. 
Auf besonders vorsichtige Dosierung bei gewissen Formen der nervösen Syphilis brauche ich hier nicht einzugehen.

Es lag mir daran, hier den Entstehungsgang der Salvarsanstörungen klarzustellen und zu zeigen, daß es heute in erster Linie an uns selbst, d. h. dem Therapeuten liegt, wenn wir die Grundlage fur Salvarsanintoxikationen schaffen.

Die von namhaften Autoren festgelegten Ursachen der Salvarsanzersetzung dürfen nicht in den Wind geschlagen und in unverantwortlicher Weise mißkreditiert werden.

Wer vor allem die Bedeutung der Wasserfehler nicht anerkennt, wird niemals in der Lage sein, das in den einzelnen Syphilisfällen notwendige Behandlungsmaß axzuwenden.

Besonders bei schwangeren Frauen bevorzuge ich heute die alleinige Neosalvarsanbehandlung als die mildeste Form der Syphilisbehandlung. Trotzdem jede einzelne Kur auf 6 Injektionen gebracht wird, habe ich bei mehr als 24 Fraven noch nie eine Störung gesehen, geschweige denn eine Unterbrechung der Gravidität.

Wie ich schon bereits oben bemerkte, können wir bei sachgemäBer Leitung der Kur wohl immer auf eine ungestörte und aussichtsvolle Therapie rechnen.

Auf die individuelle Anpassung der -einzelnen Kur an dem einzelnen Organismus, auf die genaueste Beobachtung des Patienten während der Behandlung kany ich hier natürlich nur hinweisen.

Dreuw wird indessen mit seiner Forderung, daß die Salvarsandosierung der Arsenmaximaldosis angepaßt werde, bei uns nur wenig Verständnis finden, weil wir ein derartiges minimales Quantum $(0,003-0,0045$ Neosalvarsan $)$ zu einer direkten Einverleibung in den Rückenmarkskanal benutzen. Wir haben damit bei schwerer nervöser Syphilis mehr erreicht, als durch $5-7$ Salvarsankuren à 6 Injektionen in $1 \frac{1}{2}$ bis 2 Jahren. Bei annähernd 70 Behandlungen haben wir damit keine besondere Störung gesehen.

Wenn das Salvarsan selbst ein so schweres Nervengift wäre, als es Dreuw und andere Salvarsangegner behaupten, so müBte doch bei dieser direkten Rückenmarksbehandlung das Schlimmste passieren.

Aber im Gegenteil, wir sehen eine gute therapeutische Beeinflussung aller Formen von nervöser Syphilis mit Ausnahme der allerschwersten, woselbst diese neue Behandlung nicht mehr imstande ist, die zerstörte Substanz wieder herzustellen.

Die Erfolge dieser Behandlung werfen aber nochmals ein Streiflicht auf unsere obigen Erörterungen über die bevorzugte Entwicklung der nervösen Syphilis nach unzureichender Salvarsanbehandlung. Wir wiesen darauf hin, das das SaIvarsan bei einer oberflächlichen Behandlung nicht genügend in die Rückenmarksflussigkeit gelangt und daher die dortige Infektion nur unzureichend oder gar nicht schädigte. Es war dies die Ursache, daß die Infektionsherde der Hirn- und Rückenmarksflüssigkeit bei der Rückfallbildung sich am schnellsten entwickelten und zu schweren nervösen Störungen Veranlassung gaben.

Die gleiche Unzugänglichkeit der Rückenmarksflüssigkeit für Salvarsan begegnet uns nun anch wieder im fortgeschrittenen Stadium der nervösen Syphilis. Es ist daher als ein grober Fortschritt zu begrüßen, daß wir heute gelernt haben, dea Umweg der Behandlung über die Blutbahn bei Hirn- und Rückenmarkssyphilis zu vermeiden und diese Krankheitszustände direkt anzulassen. Der erste, der diesen Weg als aussichtsreich erkannte und versuchte, war bekanntlich Wechselmann.

Die Erfolge der Salvarsanbehandlung lassen sich am Krankenmaterial der Marine besonders gut verfolgen, weil wir seit Jahren eine dienstlich geregelte Fortbeobachtung unserer Syphilisfalle besitzen. Thre Einführung erwies sich als notwendig, nicht nur urm den vermehrten Übertragungsgefahren bei dem engen Zusammenleben an Bord zu begegnen, sondern auch im Interesse der Kranken selbst, um eine gründliche Ausheilung des Leidens zu erwirken.

1. usere Behandlungsergebnisse in den ersten 3 Jahren der Salvarsanbehandlung, d. h. bis Anfang März 1913, kommen in der umstehenden labelle auf Seite 268 zum Ausdruck.

Die näheren Ausführungen zu dieser Tabelle finden sich in der in diesen Tagen erscheinenden neuesten Nummer der Münchener Medizinischen Wochenschrift.

Die berichteten Resultate zeigten sich bei einer fortlaufenden Kontrolle in klinischer Hinsicht, sowie bezüglich des vollig normalen Verhaltens der Wassermannschen Serumreaktion und der Thükenmarksflüssigkeit.

Die Steigerung der Erfolge erklärt sich aus der fortlaufenden Verbesserung der Behandlung, nicht aber aus der kürzeren. Beobachtungszeit dex Fälle aus dem letzten Berichtsjahre 1912/13.

Daß es sich ferner bei den einwandfrei verlaufenden Fällen zweifellos um eine Ausheilung des Leidens handelt, das beweisen unzweifelhaft nachstehende Beobachtungen.

1. Sind alle Falle, die wieder klinische Erscheinungen oder positive Wassermannsche Reaktion bekamen, noch im 1. Beobachtungsjahre rückfällig geworden. Das gleiche sahen wir ja auch bei der mäBigen Salvarsanbehandlung Ravauts, der in über $80 \%$ der Fälle klinische Erscheinungen noch im 1. Jahre wiederkehren sah.

2. Zeigen $90 \%$ aller Syphilisrückfälle nach Salvarsanbehandlung im frischen Stadium Veränderungen der Rückenmarksflüssigkeit, die in unseren Fällen fehlen, trotz mehriacher Untersuchung in einjährigen Zeitabständen. Es ist damit auch eine syphilitische Späterkrankung am Nervensystem auszuschlieBen. 


\begin{tabular}{|c|c|c|c|c|c|c|c|c|c|c|c|c|c|c|c|}
\hline \multirow{3}{*}{$\begin{array}{l}\text { Berichts- } \\
\text { zeitraum }\end{array}$} & \multicolumn{6}{|c|}{ Frische Syphilisfälle } & \multirow{2}{*}{\multicolumn{3}{|c|}{$\begin{array}{c}\text { Altere Syphilisfälle } \\
\text { mit sekundären } \\
\text { Erscheinungen }\end{array}$}} & \multirow{2}{*}{\multicolumn{3}{|c|}{$\begin{array}{c}\text { A.ttere Syphilisfälle } \\
\text { mit tertiären } \\
\text { Erscheinungen }\end{array}$}} & \multirow{2}{*}{\multicolumn{3}{|c|}{$\begin{array}{l}\text { Syphilisfälle } \\
\text { ohne sichtliche } \\
\text { Erscheinungen }\end{array}$}} \\
\hline & \multicolumn{3}{|c|}{$\begin{array}{l}\text { Noch ohne Allge- } \\
\text { meinerscheinungen }\end{array}$} & \multicolumn{3}{|c|}{$\begin{array}{l}\text { Bereits mit Allge- } \\
\text { meinerscheinungen }\end{array}$} & & & & & & & & & \\
\hline & 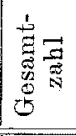 & 䨌 & 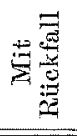 & 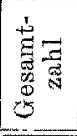 & 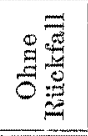 & 吾 & 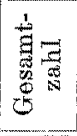 & 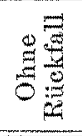 & 焉 & 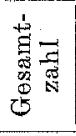 & 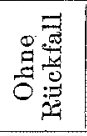 & 焉 & 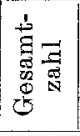 & 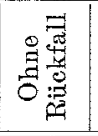 & 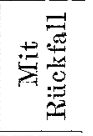 \\
\hline $\begin{array}{c}\text { Juli } 1910- \\
\text { März } 1911 \\
\text { absol. Zahl } \\
\text { Prozentsatz }\end{array}$ & $5 \mathrm{R}$ & $\begin{array}{c}34 \\
85 \% \\
\text { einfekti }\end{array}$ & $\begin{array}{c}5 \\
13 \% \\
\text { onen }\end{array}$ & 38 & $\begin{array}{c}20 \\
52,6 \%\end{array}$ & $\begin{array}{c}18 \\
47,4 \%\end{array}$ & 8 & $\begin{array}{c}2 \\
25 \%\end{array}$ & $\begin{array}{c}6 \\
75 \%\end{array}$ & 9 & $\begin{array}{c}5 \\
550 \%\end{array}$ & $\begin{array}{c}4 \\
45 \%\end{array}$ & 20 & $\begin{array}{c}12 \\
55^{0 / 0}\end{array}$ & $\begin{array}{c}8 \\
45 \%\end{array}$ \\
\hline $\begin{array}{c}\text { Marz } 1911-\text { - } \\
\text { Marz } 1912 \\
\text { absol. Zahl } \\
\text { Prozentsatz }\end{array}$ & $7 \mathrm{R}$ & $\begin{array}{c}65 \\
89 \% \\
\text { einfekti }\end{array}$ & $\begin{array}{c}8 \\
11 \% \\
\text { onen }\end{array}$ & 78 & $\begin{array}{c}67 \\
87 \%\end{array}$ & $\begin{array}{c}10 \\
13 \%\end{array}$ & 17 & $\begin{array}{c}12 \\
70 \%\end{array}$ & $\begin{array}{c}5 \\
30 \%\end{array}$ & 12 & $\begin{array}{c}11 \\
92 \%\end{array}$ & $\begin{array}{c}1 \\
8 \%\end{array}$ & 32 & $\begin{array}{c}28 \\
87,5 \%\end{array}$ & $\begin{array}{c}4 \\
12,5 \%\end{array}$ \\
\hline $\left.\begin{array}{c}\text { Marz } 1912-\} \\
\text { März } 1913\end{array}\right\}$ & $1 \mathrm{r}$ & $\begin{array}{c}89 \\
97 \% \\
\text { einfekti }\end{array}$ & $\begin{array}{c}3 \\
3 \% \\
10 n\end{array}$ & $3 \mathrm{R}$ & $\begin{array}{c}66 \\
94 \% \\
\text { einfekt }\end{array}$ & $\begin{array}{c}4 \\
6 \% \\
6 n e n\end{array}$ & 11 & $\begin{array}{c}10 \\
90 \%\end{array}$ & $\begin{array}{c}1 \\
10 \%\end{array}$ & 8 & $\begin{array}{c}8 \\
100 \%\end{array}$ & - & 43 & $\begin{array}{c}42 \\
98 \%\end{array}$ & $\begin{array}{c}1 \\
2 \%\end{array}$ \\
\hline
\end{tabular}

3. Ist in 17 Fällen, die bis über 3 Jahre hin einen völlig einwandfreien Verlauf nach Abschluß der Behandlung aufgewiesen haben, die weder klinisch, noch im Verhalten ihrer Serumreaktion, noch in der Rückenmarksflüssigkeit einen Anhalt für das Fortbestehen der syphilitischen Erkrankung darboten, eine Wiederansteckung mit frischer Syphilis erfolgt, die sich in gleicher Weise entwickelte, als ob die Fälle niemals mit Syphilis infiziert gewesen wären. Es ist dies ein noch nie dagewesener Vorgang, wie er in den vielen Jahren der alleinigen Quecksilberbehandlung nur in einem Falle nach sehr ausgiebigen. Kuren von uns beobachtet worden ist.

4. Sind nach Abschluß der Behandlung nie Fehlgeburten beobachtet worden. Es ist bereits jetzt eine gröfere Anzahl gesunder Kinder vorhander mit einem Alter bis zu 3 Jahren.

Wir befinden uns auch in völliger t̛bereinstimmung mit den Ansichten Neißers, welcher nach einem derart langen einwandfreien Verlauf der Syphilisfälle in klinischer und serologischer Hinsicht und in der Rückenmarksflüssigkeit eine völlige Ausheilung der Syphilis als zweifellos bezeichnet hat.

Die berichteten Exfolge der Salvarsanbehandlung sind ein glänzender Beweis für die außerordentlichen Heilkräfte dieser modernen Syphilisbehandlung. Sie übertreffen besonders noch dadurch alle Beobachtungen über die Erfolge der früheren üblichen Behandlungsmethoden, weil sie in so enorm kurzer Zeit erreicht werden konnten.

Auch der Fermerstehende wird und muß es empfinder, daß wir mit dem Ausbau der Salvarsanbehandlung an einem bedeutsamen Wende- punlto in dex Syphilistherapie, wie in der Geschichte der Syphilis überhaupt angelangt sind, und dal wir dem Erfinder des Salvarsans für alle Zeiten unseren größten Dank schuldig sind.

Müssen wir es nicht als einen ganz naturgemäfien Vorgang betrachten, daß der Entwicklungsgang einer so enorm wirksamen Behandlung, als die wir die Salvarsambehandlung kennen gelernt habon, vicht ohne einige Opfer vor sich gehen konnte?

Tch bezweille zwar sehr, daß die Dreuwschen Angaben über die Zahl der Todesfälle, die in der Tat dem Salvarsan selbst zur Last fallen, zutreffend sind, ich muß aber nach unseren Erfahrungen über die Leistungsfähigkeit der Methode vor der Offentlichkeit feststellen, daß die Anzahl der unglücklichen Ausgänge gegenüber den Segnungen der Behandlung, die sich bei uns an mehr als 1200 Fällen fortlaufend offenbaren, überhaupt nicht ins Gewicht fällt.

Daß wir ferner bei der Marine in der Lage waren, unseren Patienten die Vorteile der Salvarsanbehandlung zukommen zu lassen, verdanken wir allein der weitschauenden Fürsorge und Unterstützung unserer Medizinalbehörde, welche einer Schöpfung Ehrlichs unbedingtes Vertrauen entgegenbrachte.

\section{Paul Ehrlich als Chemiker.}

Von Dr. L. Benda, Frankfurt a. M.

"Corpora non agunt nisi fixata." Wie einfach klingen diese Worte, die Ehrlich zum Grundsatz seiner chemotherapeutischen Studien gemacht 